\title{
Circum-nuclear molecular disks: role in AGN fueling and feedback
}

\author{
Francoise Combes \\ Observatoire de Paris, LERMA, Collège de France, CNRS, \\ PSL University, Sorbonne University, UPMC, Paris \\ email: francoise.combes@obspm.fr
}

\begin{abstract}
Gas inflows fueling AGN are now traceable at high-resolution with ALMA and NOEMA. Dynamical mechanisms are essential to exchange angular momentum and drive the gas to the super-massive black hole. While at 100pc scale, the gas is sometimes stalled in nuclear rings, recent observations reaching 10pc scale (50mas), inside the sphere of influence of the black hole, may bring smoking gun evidence of fueling, within a randomly oriented nuclear molecular disk. AGN feedback is also observed, in the form of narrow and collimated molecular outflows, which point towards the radio mode, or entrainment by a radio jet. Precession has been observed in a molecular outflow, indicating the precession of the radio jet. One of the best candidates for precession is the Bardeen-Petterson effect at small scale, which exerts a torque on the accreting material, and produces an extended disk warp. The misalignment between the inner and largescale disk, enhances the coupling of the AGN feedback, since the jet sweeps a large part of the molecular disk.
\end{abstract}

Keywords. galaxies: active, galaxies: general, galaxies: nuclei, galaxies: Seyfert, galaxies: spiral

\section{Introduction}

It is now well established that there exists a tight relation between the mass of the central supermassive black hole, and the bulge mass, or central velocity dispersion, which has been interpreted as a co-evolution of galaxies and black holes (e.g., Kormendy and Ho 2013: Heckman and Best 2014). This co-evolution might be due to a common feeding mechanism, either through mergers or cosmic gas accretion followed by secular evolution, as recenty reviewed by Storchi-Bergmann and Schnorr-Müller (2019), and/or to AGN feedback mechanisms, regulating the star formation in the galaxy host (e.g., Fabian 2012, Morganti and Oosterloo 2018).

The new frontier in this domain is to understand in more details the feeding and feedback mechanisms at the highest possible resolution, in the complex circumnuclear region, surrounding the black hole, with the help of multi-wavelength observations (RamosAlmeida and Ricci 2017). A new view is emerging, where the absorbing material is not due to the long-expected dusty torus (Hönig 2019). VLT Interferometer (VLTI) observations showed that the dust on parsec scales is not mainly in a thick torus, but instead in a polar structure, forming like a hollow cone, perpendicular to a thin disk (e.g., Asmus et al. 2016: Asmus 2019). The circumnuclear region, as a transition between the Broad Line Region (BLR) of the accretion disk, and the Narrow Line Region (NLR), is complex and clumpy, and contains both inflowing material in a thin disk, where millimeter lines have been found, with also $\mathrm{H}_{2} \mathrm{O}$ masers, and an outflowing component, in the perpendicular direction (e.g., Cicone et al. 2014, Garcia-Burillo et al. 2016).

In the following, I review ALMA observations at high-angular resolution of the molecular gas, revealing nuclear trailing spiral features, that explain the feeding of the central 

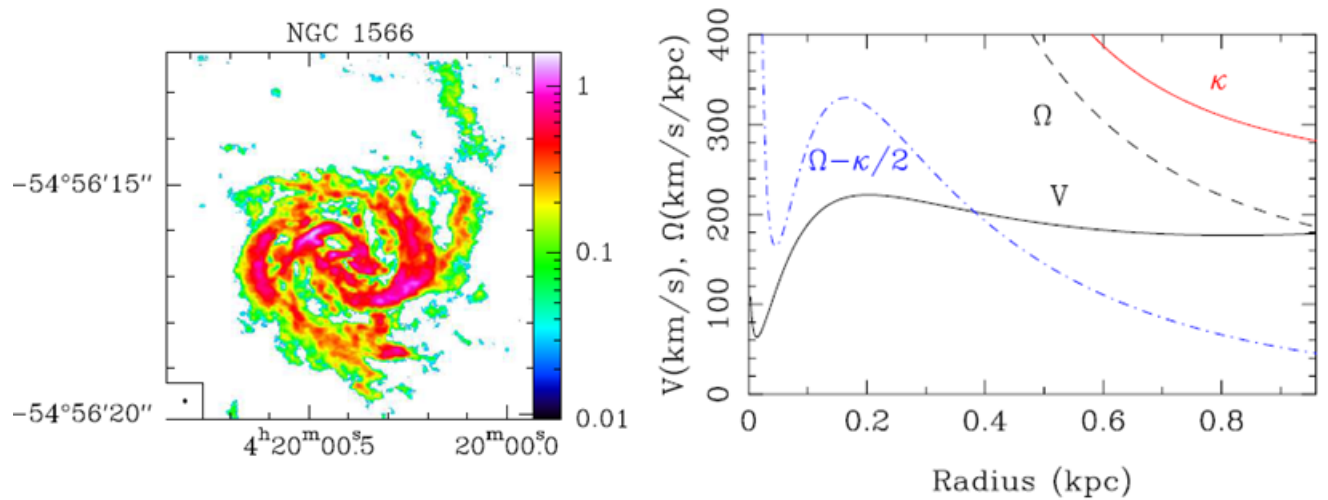

Figure 1. ALMA observations of the Seyfert-1 galaxy NGC 1566. Left is a zoomed 8"x8" region of the $\mathrm{CO}(3-2)$ intensity map, showing the nuclear trailing spiral $(1 "=35 \mathrm{pc})$. Right, is the model rotation curve from NIR images, represented schematically, with the circular velocity (black) epicyclic frequency $\kappa$ (red), and corresponding $\Omega-\kappa / 2$ curve (blue) within the central kpc. The contribution of a super-massive black hole in the nucleus with $\mathrm{MBH}=8.3 \quad 10^{6} \mathrm{M}_{\odot}$ has been included. From Combes et al. (2014, 2019).

black hole, through exchange of angular momentum. ALMA observations have also revealed outflows, some being extremely collimated in a molecular jet. These outflows must be due to the radio mode of AGN feedback, even when no radio jet has yet been detected. ALMA has also revealed in most nearby Seyferts the existence of molecular circum-nuclear disks, misaligned with the large-scale disks, and with decoupled kinematics. We identify these parsec-scale structures as molecular tori, able to obscure the central accretion disks. Several mechanisms are reviewed to explain the misalignments.

\section{Feeding the monster}

To fuel the central black hole, the main problem is to transfer the angular momentum of the gas outwards. This can be done by the gravity torques exerted by bars on the gas in spiral arms (Garcia-Burillo et al. 2005). Torques are positive outside corotation, and the gas is driven outward to accumulate in a ring at the Outer Lindblad Resonance (OLR). Inside corotation, torques are negative, and gas is driven inward, to pile up in a nuclear ring at the Inner Lindblad resonance (ILR).

What happens inside the ILR depends on the winding sense of orbits there. The gas is orbiting in elliptic streamlines, which gradually tilt by $90^{\circ}$ at each resonance and wind up in spiral structures. The precession rate of these elliptical orbits is equal to $\Omega-\kappa / 2$, with $\Omega$ the rotation frequency $=\mathrm{V} / \mathrm{r}$, and $\kappa$ the epicyclic frequency. Usually, inside ILR, and far from the black hole, $\Omega-\kappa / 2$ increases with radius, and the spiral is leading. The torque of the bar is positive, and the gas is driven back to the ILR. But near the massive black hole, the precessing frequency $\Omega-\kappa / 2$ is decreasing with radius, and the spiral is trailing. The gas can then fuel the AGN (Buta \& Combes 1996).

ALMA has the resolution to enter the sphere of influence of the black hole, and a trailing nuclear spiral was first seen in NGC 1566 (Combes et al. 2014). This nuclear spiral is located well inside the $\mathrm{r}=400 \mathrm{pc}$ ring, corresponding to the ILR of the bar. Fig. 1 shows the nuclear spiral in the $\mathrm{CO}(3-2)$ line (left), and at right the rotation curve and corresponding frequencies, derived from the stellar potential traced by near-infrared images. The precessing rate $\Omega-\kappa / 2$ increases towards the center, inside $50 \mathrm{pc}$, due to 

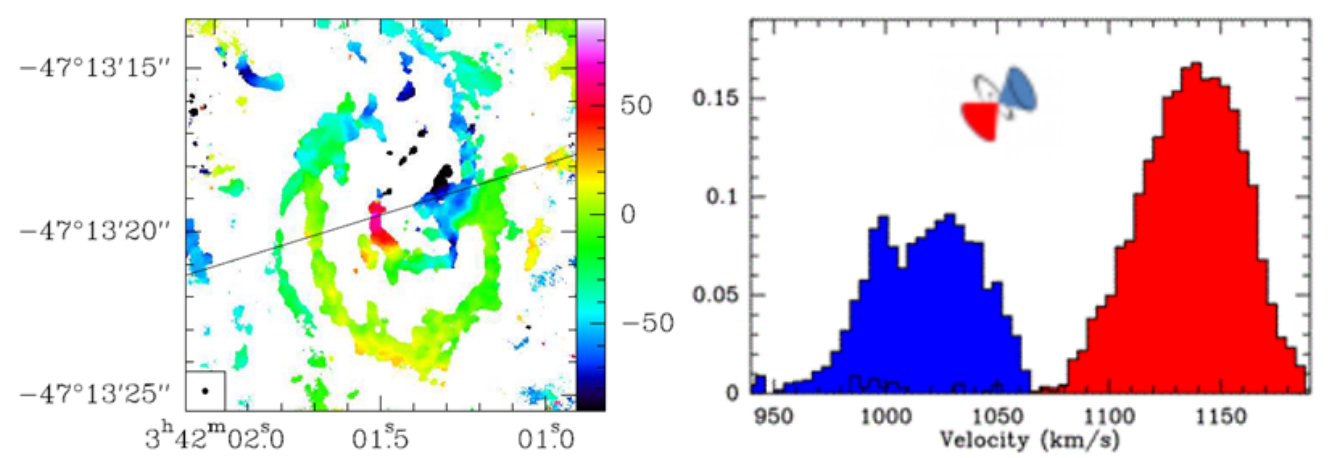

Figure 2. ALMA CO(3-2) observations of the Seyfert-2 NGC 1433: Left, the velocity field, with the color bar labelled in $\mathrm{km} / \mathrm{s}$; The thin line indicates the minor axis $\left(\mathrm{PA}=109^{\circ}\right)$. Right, spectrum summing the blue and red-shifted components close to the center, along the minor axis. The systemic velocity is $\mathrm{V}_{s y s}=1075 \mathrm{~km} / \mathrm{s}$. The mass in the outflow is $3.610^{6} \mathrm{M}_{\odot}$. From Combes et al. (2013, 2019).

a black hole of mass $8.3 \quad 10^{6} \mathrm{M}_{\odot}$. Such trailing nuclear spirals have been found also in NGC 613 (Audibert et al. 2019) and in NGC 1808 (Audibert et al. 2020, in prep.).

\section{AGN feedback: jets and winds}

Molecular outflows are now commonly observed as AGN feedback (Cicone et al. 2014). If NGC 1566 does not reveal any outflow, both inflow and outflow can be observed simultaneously, as in NGC 613 , where a very short (23pc) and small velocity $(300 \mathrm{~km} / \mathrm{s})$ outflow is detected on the minor axis, parallel to the VLA radio jet Audibert et al. 2019). A very small molecular outflow is also seen in NGC 1433, along the minor axis, cf Fig. 2. This might be the smallest molecular outflow in a nearby Seyfert galaxy, and could be associated to a past radio jet (e.g., Combes et al. 2013 Smajic et al. 2014).

In these nearby low-luminosity AGN, which accrete far below the Eddington limit, the main mechanism to drive molecular outflows is the radio mode, i.e. entrainement by the radio jets. In some more luminous cases, where $\mathrm{L}$ approaches $\mathrm{L}_{E d d} / 100$, there could be both the radio mode, and winds generated by radiation pressure (either in the ionized gas, or on dust). This might be the case of the prototypical Seyfert-2 NGC 1068, where there is clearly a molecular outflow parallel to the radio jet, sweeping part of the galactic disk (Garcia-Burillo et al. 2014). The jet is not perpendicular to the plane, due to the misalignment of the accretion disk with the plane. The ALMA observations of the various $\mathrm{CO}$ rotational lines reveal clearly a molecular torus, almost edge-on, and a molecular flow in the perpendicular direction, aligned with the polar dust. The molecular disk appears warped and tilted with respect to the $\mathrm{H}_{2} \mathrm{O}$ maser disk (Garcia-Burillo et al. 2016 ).

The lenticular galaxy NGC 1377 is an exceptional case, with a very thin and highy collimated molecular outflow, in the absence of any detected radio jet (Aalto et al. 2016). The molecular outflow changes sign along the flow, on each side of the galaxy. This means that the jet is almost in the plane of the sky, and that a slight precession of only $10^{\circ}$ is able to tip the jet from redshifted to blue-shifted and back. Such a precession is observed in micro-quasars jets in the Milky Way, for instance SS433 (Mioduszewski et al. 2005). But this can be attributed to the companion star. Here there must exist another origin of the precesion, which could be relativistic (see next section). 
A precessing molecular outflow model is compatible with the data (Aalto et al. 2016). The flow is launched close to the center $(\mathrm{r}<10 \mathrm{pc})$. A radio jet must exist at a low level, or has existed in a recent past.

\section{Molecular tori: misalignment}

With the high spatial resolution of ALMA, it was possible to unveil circumnuclear disks in the CO emission, towards nearby Seyferts. These happen to be misaligned to the large-scale disks, and kinematically decoupled. We call them molecular tori, they exist in 7 out of the 8 cases observed (Combes et al. 2019). The average radius of the molecular tori is $18 \mathrm{pc}$, with a median at $21 \mathrm{pc}$. Their average molecular mass is $\mathrm{M}\left(\mathrm{H}_{2}\right)=1.410^{7}$ $\mathrm{M}_{\odot}$, and in average their inclination on the plane of the sky is $29^{\circ}$ different from their galactic disk.

These molecular tori are clearly within the sphere of influence of their black holes, and can serve to measure their mass, provided that their inclination is sufficient Combes et al. 2019). This has been done also for more massive early-type galaxies, by the WISDOM project (Davis et al. 2018 ).

We can invoke at least three mechanisms of misalignment between the large-scale galactic disks and the molecular tori and/or accretion disks. One of them is the radiationdriven warping instability (Pringle 1996). A tilted optically thick disk, which absorbs the radiation from the central $\mathrm{AGN}$, receives in each point some momentum from the radiation, but no torque, because of the radial direction. But then it re-radiates perpendicularly to its orientation, and this produce torques, which maintain and amplify the warping. Assuming the luminosity is powered by accretion eliminates the unknown viscosity parameter $\alpha$. The instability occurs for radii $\mathrm{R}>0.1 \mathrm{pc} \mathrm{M}_{B H} /\left(10^{8} \mathrm{M}_{\odot}\right)$. The efficiency of the mechanism was tested by simulations, both in the case of retrograde and prograde precession with respect to the disk rotation (Maloney and Begelmann 1997). A second meschanism is the magnetic instability, and consequent torques but compatibility with AGN observations is contrived, it is more adapted to accretion disks around magnetic stars (Pfeiffer \& Lai 2004).

A third mechanism uses the Bardeen-Petterson effect (Bardeen and Petterson 1975), due to Lense-Thirring precession. The accretion disk has a random orientation, generally not aligned with the black hole spin. The relativistic frame dragging effect induces a precession, which tends to align the inner parts of the accretion disk with the black hole equator. The disk develops a warping up to distances $10^{2}$ to $10^{4}$ Schwarzschild radius $\mathrm{R}_{s}$. The precession of the disk and its warp can be seen from inner to outer disk, up to 1 pc $\mathrm{M}_{B H} /\left(10^{9} \mathrm{M}_{\odot}\right)$. According to the amplitude of viscosity, one can distinguish two regimes: the diffusion, when $\alpha>\mathrm{H} / \mathrm{R}$, where $\mathrm{H}$ is the height of the disk, and the regime of bending waves, when $\alpha<\mathrm{H} / \mathrm{R}$ (Papaloizou \& Pringle 1983). In the first case, the disk is warping smoothly and continuously, while in the second case, the disk can break in several rings, with different inclinations and precessing rates. Then the various rings, with differential precession, collide, and drive the gas to fuel the AGN more quickly. This regime has been simulated by Nealon et al. (2015). Some works found that the alignment of accretion disks with the black hole equator, through the Bardeen-Petterson effect, was inefficient (Zhuravlev et al. 2014 ; Banerjee et al. $2019 \mathrm{a}$ b). More precisely, according to some viscous parameters (parallel of perpendicular to the disk), and viscosity generated by magnetized turbulence, the disk near the black hole can retain its inital inclination, instead of aligning, cf. Fig. 3. Then precessing jets can be launched, perpendicular to the disk, but not aligned to the black hole spin (Liska et al. 2018,2019 ). 

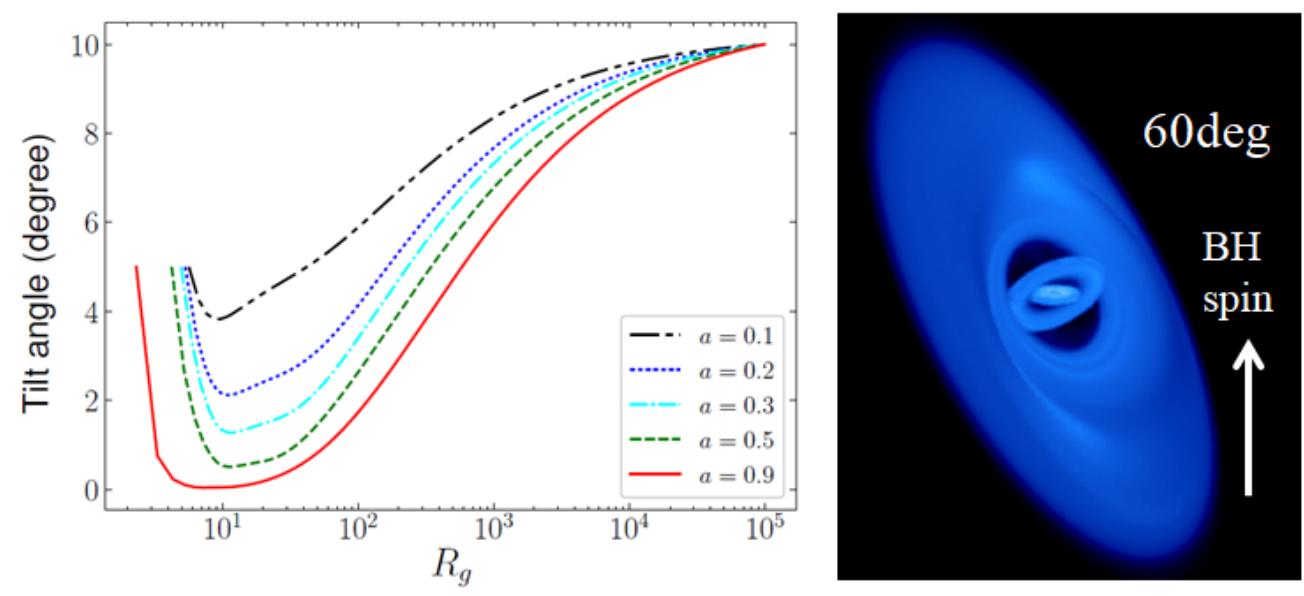

Figure 3. Misalignment of the accreting material near a black hole: Left: radial profiles of the disk tilt-angle (with respect to the black hole spin), for several values of the parameter a, dimensionless angular momentum $J$ of the black hole $M, 0<\mathrm{a}<1\left(\mathrm{a}=\mathrm{c} J /\left(\mathrm{G} M^{2}\right)\right)$. The initial tilt-angle is $5^{\circ}$. The radius is in unit of the gravitational radius $\mathrm{R}_{g}=\mathrm{G} M / \mathrm{c}^{2}$. From Banerjee et al. (2019a). Right: Simulation of the Bardeen-Petterson effect, in a disk initially inclined by $60^{\circ}$ with respect to the black hole spin. The Lense-Thirring precession causes the disk to break in a few tilted rings. Adapted from Nealon et al. (2015).

A manifestation of these warping instabilities is the observation of the warped maser disks. Water masers in the prototypes NGC 4258 (Herrnstein et al. 1999) and NGC 1068 (Gallimore et al. 2004) are detected on $0.3-0.8$ pc warped discs. These observations are best represented by the Lense-Thirring effect and/or the radiation driven warps. These perturbations also heat the disk. The fitting of the observations has been done for NGC 4258 by Martin (2008), and for NGC 1068 by Caproni et al. (2006). In NGC 1068, where the Bardeen-Petterson mechanism gives the best fit with the observations, the disk is aligned with the black hole spin, until the radius $\mathrm{R}_{B P}=10^{-5}$ to $10^{-4} \mathrm{pc}$, then warps. For one of the best fit models the alignment time-scale is $7580 \mathrm{yr}$, the misalignment angle $40^{\circ}$ and the velocity of the jet $0.17 \mathrm{c}$. The shape and precession of the pc and kpc-scale jet is also fitted, following Wilson and Ulvestad (1987), in addition to the warped $\mathrm{H}_{2} \mathrm{O}$ maser disk by Gallimore et al. (2004).

How can the gas accreted from the galactic disk be so misaligned with the disk itself? First, the potential in the center is almost spherical, and the disk very thick with respect to the parsec-scales in question, and second, star formation feedback constantly ejects some gas out of the plane, which rains down in a fountain effect at a random orientation, sometimes in a polar ring (Renaud et al. 2015 , Emsellem et al. 2015$)$.

\section{Summary}

Thanks to the high resolution provided by ALMA on the molecular gas, it is now possible to better understand the AGN fueling mechanisms. If at large scale, the primary bars can drive the gas towards the 100pc scales, in ILR rings, the nuclear bars act on a trailing nuclear spiral to drive the gas towards the black hole, when the circumnuclear gas enters its sphere of influence. Inside the nuclear spiral, ALMA has revealed the existence of morphologically and kinematically decoupled circum-nuclear disks, or molecular tori.

In some less frequent cases, we can see both AGN fueling, and molecular outflows, 
through AGN feedback. This can occur via entrainment by radio jets, or through disk winds (or both). The radio mode is distinguished by extremely thin and collimated molecular jets, sometimes precessing, as are the entraining radio jets.

To explain the precession, and the misalignment of the circum-nuclear disks, we can invoke at small scale the Bardeen-Petterson effect, which produces torques in the accreting material, to align it with the black hole spin, and then induces a warping up to a fraction of parsec-scale. The black hole is likely to be fueled via several accretion episodes, coming from the large-scale galacic disk, but also the fountain gas, ejected above the plane through supernovae feedback.

\section{References}

Aalto, S., Costagliola, F., Muller, S. et al. 2016, A\& $A$, 590, A73

Asmus, D., 2019, MNRAS, 489, 2177

Asmus, D., Hönig, S. F., Gandhi, P. 2016, Ap. J., 822, 109

Audibert, A., Combes, F., Garcia-Burillo, S. et al. 2019, A\& A, 632, A33

Banerjee, S., Chakraborty, C., Bhattacharyya, S. 2019b, MNRAS, 487, 3488

Banerjee, S., Chakraborty, C., Bhattacharyya, S. 2019a, Ap. J., 870, 95

Bardeen, J.M., Petterson, J.A. 1975, Ap. J., 195, L65

Buta, R., \& Combes, F. 1996, Fund. Cosmic Phys., 17, 95.

Caproni, A., Abraham, Z., Mosquera Cuesta, H.J. 2006, Ap. J., 638, 120

Cicone, C., Maiolino, R., Sturm, E. et al. 2014, A\&A, 562, A21

Combes, F., Garcia-Burillo, S., Casasola, V. et al. 2013, A\&A, 558, A124

Combes, F., Garcia-Burillo, S., Casasola, V. et al. 2014, $A \mathscr{E} A$, 565, A97

Combes, F., Garcia-Burillo, S., Audibert, A et al. 2019, A\&A, 623, A79

Davis, T. A., Bureau, M., Onishi, K., et al. 2018, MNRAS, 473, 3818

Emsellem, E., Renaud, F., Bournaud, F. et al. 2015, MNRAS, 446, 2468

Fabian, A.C. 2012, ARAA, 50, 455

Gallimore, J.F., Baum, S.A., O’Dea, C.P. 2004, Ap. J., 613, 794

Garcia-Burillo, S., Combes, F., Schinnerer, E. et al. 2005, A\&A, 441, 1011

Garcia-Burillo, S., Combes, F., Usero, A. et al. 2014, A $6 A$, 567, A125

Garcia-Burillo, S., Combes, F., Ramos Almeida, C. et al. 2016, Ap. J., 823, L12

Heckman, T. M., Best, P. N. 2014, ARAA, 52, 589

Herrnstein, J.R., Moran, J.M., Greenhill, L.J. et al. 1999, Nature, 400, 539

Hönig, S.F. 2019, Ap. J., 884, 171

Kormendy, J., Ho, L. C. 2013, ARAA, 51, 511

Liska, M., Hesp, C., Tchekhovskoy, A. et al. 2018, MNRAS, 474, L81

Liska, M., Tchekhovskoy, A., Ingram, A. et al. 2019, MNRAS, 487, 550

Maloney, P.R., Begelman, M.C. 1997, Ap. J., 491, L43

Martin, R. 2008, MNRAS, 387, 830

Mioduszewski A. J., Dhawan, V., Rupen, M. P. 2005, ASPC, 340, 281

Morganti, R., Oosterloo, T. 2018, A\&ARv, 26, 4

Nealon, R., Price, D.J., Nixon, C.J.2015, MNRAS, 448, 1526

Papaloizou, J. C. B., Pringle, J. E.1983, MNRAS, 202, 1181

Pfeiffer, H. P., Lai, D.2004, Ap. J., 604, 766

Pringle, J. E.1996, MNRAS, 281, 357

Ramos-Almeida, C., Ricci, C. 2017, NatAs, 1, 679

Renaud, F., Bournaud, F., Emsellem, E. et al. 2015, MNRAS, 454, 3299

Smajic, S., Moser, L., Eckart, A. et al. 2014, A\&A, 567, A119

Storchi-Bergmann, T, Schnorr-Müller, A. 2019, NatAs, 3, 48

Wilson, A.S., Ulvestad, J.S. 1987, Ap. J., 319, 105

Zhuravlev, V.V., Ivanov, P.B., Fragile, P.C. et al. 2014, Ap. J., 796, 104 